\title{
PRECAUÇÕES ESPECÍFICAS PARA EVITAR A TRANSMISSÃO DE MICRORGANISMOS: DESENVOLVIMENTO E VALIDAÇÃO DE ROTEIRO EDUCACIONAL*
}

\author{
Luize Fábrega Juskevicius ${ }^{1}$, Maria Clara Padoveze ${ }^{2}$
}

\begin{abstract}
RESUMO: O objetivo deste estudo foi desenvolver e validar o conteúdo do roteiro educativo para indivíduos em precauções específicas para evitar a transmissão de microrganismos. Estudo metodológico, desenvolvido em três fases,em dois hospitais (público e privado) na cidade de São Paulo, no período de maio a julho de 2015, utilizando o referencial teórico de Vulnerabilidade. Na primeira fase, aplicou-se questionário para captação das percepções dos indivíduos adultos sobre precauções específicas. Na segunda fase, elaborou-se o roteiro educativo. Na terceira fase, foi submetido a especialistas em precauções específicas e vulnerabilidade para validação de conteúdo. Foram entrevistados39 indivíduos em média sete dias após a instituição das precauções específicas, 32 (82\%) em precaução para contato. O roteiro foi desenvolvido para proporcionar maior conhecimento nos aspectos usualmente negligenciados pelos profissionais e estimular o cuidado centrado no indivíduo. Todos os itens tiveram um índice de validade de conteúdo acima de $75 \%$. O roteiro apresenta potencial para instrumentalizar profissionais na elaboração de ações educativas para pacientes adultos em precauções específicas.
\end{abstract}

DESCRITORES: Vulnerabilidade em saúde; Precauções universais; Acesso à informação; Participação do paciente; Educação em saúde.

\section{SPECIFIC PRECAUTIONS FOR AVOIDING MICROORGANISM TRANSMISSION: DEVELOPMENT AND VALIDATION OF AN EDUCATIONAL GUIDE}

\begin{abstract}
This study aimed to develop and validate the content of the educational guide for individuals under specific precautions for avoiding microorganism transmission. It is a methodological study performed in three phases, in two hospitals (public and private) in the city of São Paulo, from May to July 2015, using the theoretical framework of Vulnerability. In the first phase, a questionnaire was applied for capturing the adult individuals' perceptions regarding specific precautions. In the second phase, the educational guide was elaborated. In the third phase, the guide was submitted to specialists in specific precautions and vulnerability for content validation. Interviews were held with 39 individuals, an average of seven days after the institution of the specific precautions, 32 $(82 \%)$ of whom were under contact precautions. The guide was developed to provide better knowledge of aspects usually neglected by professionals, and to encourage person-centred care. All items had a content validity index of above $75 \%$. The guide presents potential to support professionals in the development of educational actions for adult patients subject to specific precautions.

DESCRIPTORS: Vulnerability in health; Universal precautions; Access to information; Patient participation; Education, health.
\end{abstract}

\section{PRECAUCIONES ESPECÍFICAS PARA EVITAR LA TRANSMISIÓN DE MICROORGANISMOS: DESARROLLOY VALIDACIÓN DE GUION EDUCACIONAL}

RESUMEN: La finalidad de este estudio fue desarrollar y validar el contenido de guion educativo de precauciones específicas con fines de evitar la transmisión de microorganismos. Es un estudio metodológico, hecho entres fases, en dos hospitales (público y privado) en la ciudad de São Paulo, en periodo de mayo a julio de 2015, utilizándose referencial teórico de Vulnerabilidad. Fue aplicado cuestionario para captación de percepciones de los individuos adultos acerca de precauciones específicas en la primera fase. En la segunda fase, se elaboró guion educativo. En la tercera fase, ello fue sometido a especialistas en precauciones específicas y vulnerabilidad para validación de contenido. Fueron entrevistados 39 individuos, en media siete días después de la institución de las medidas preventivas específicas, $32(82 \%)$ en precaución para contacto. El guion fue desarrollado para proporcionar más conocimiento acerca de los aspectos generalmente negligenciados por los profesionales y estimular el cuidado centrado en el individuo. Todos los puntos tuvieron índice de validad de contenido superior a75\%. El guiones una potencial herramienta a los profesionales en la elaboración de acciones educativas para pacientes adultos en precauciones específicas.

DESCRIPTORES: Vulnerabilidad en salud; Precauciones universales; Acceso a la información; Participación del paciente; Educación en salud.

\footnotetext{
*Artigo extraído da dissertação intitulada: "Precauções específicas para evitar a transmissão de microrganismos: elaboração e validação de roteiro para contribuir na redução da vulnerabilidade individual". Escola de Enfermagem da Universidade de São Paulo, 2016.
}

${ }^{1}$ Enfermeira. Mestre em Ciência da Saúde. Enfermeira do Controle de Infecção. Escola de Enfermagem da Universidade de São Paulo. São Paulo, SP, Brasil.

${ }^{2}$ Enfermeira. Doutora em Clínica Médica. Docente de Enfermagem em Saúde Coletiva. Escola de Enfermagem da Universidade de São Paulo. São Paulo, SP, Brasil. 


\section{INTRODUÇÃO}

A propagação das infecções pode ocorrer devido ao não cumprimento das precauções padrão (PP) e precauções específicas (PE) por parte dos profissionais da assistência a saúde (PAS) e visitantes. Estudos demonstraram baixa adesão às medidas de precaução pelos PAS, o que pode ter relação com os aspectos do comportamento humano, como a não percepção do risco e a subestimação do papel individual nas taxas de infecções relacionadas à assistência à saúde (IRAS) ${ }^{(1)}$.

Com relação aos indivíduos e familiares, a orientação é não raro fragmentada e sem esclarecer aspectos relevantes das precauções, restringindo-se apenas em indicar o que deve ou não ser feito. Isto pode ter como consequência as falhas na adesão a procedimentos pela incompreensão do processo como um todo ${ }^{(2)}$.

As PE criam barreiras físicas e sociais, o que pode aumentar os níveis de estresse no indivíduo que está submetido a esta condição. Neste contexto, os PAS precisam atender tanto às necessidades do indivíduo sob seus cuidados como lidar com seus próprios medos de contrair uma doença ${ }^{(3)}$. O acesso à informação qualificada tem potencial para diminuir os impactos psicológicos negativos relacionados às PE.

Indivíduos em PE podem estar mais vulneráveis à ocorrência de eventos adversos, uma vez que os PAS entram menos em seus quartos e suas visitas tem um tempo reduzido em comparação com os que não estão em $\mathrm{PE}^{(3-4)}$. Os eventos adversos relacionados às $\mathrm{PE}$ caracterizam-se por possuir componentes amplos e inter-relacionados, que não podem ser avaliados e tratados de forma isolada. Desta forma, considera-se importante outras formas de refletir sobre as intervenções de saúde.

A partir da década de 1980, teóricos em saúde coletiva propuseram o conceito de Vulnerabilidade para ser utilizado como quadro de referência para apoiar o manejo de agravos em saúde. Este conceito nasceu e se corporificou no enfoque ao vírus da imunodeficiência humana (HIV), almejando o alcance de resultados mais efetivos em saúde e com redução do estigma associado a doença ${ }^{(5)}$. Isto permitiu uma nova abordagem pelos profissionais de saúde para elaboração de propostas de intervenção, contribuindo para os avanços obtidos ${ }^{(6)}$.

O conceito de vulnerabilidade é composto por três dimensões, a saber: individual, social e programática. Na perspectiva conceitual estas dimensões são de tal forma interconectadas que são efetivamente indissociáveis.A dimensão individual está relacionada com o comportamento, a experiência de vida no contexto do seu meio social, bem como os graus de consciência sobre determinada situação e o decorrente empoderamento para a sua transformação ${ }^{(7)}$.

O conceito de vulnerabilidade possui potencial para apoiar as abordagens que visam a reduzir os eventos adversos relacionados às PE, na medida em que oferta um suporte teórico ampliado, menos tecnicista e mais centrado nas necessidades dos indivíduos. Entretanto, em uma revisão de literatura, não identificamos estudos que se dedicaram a explorar o uso do conceito de Vulnerabilidade para apoiar as ações de prevenção da transmissão de microrganismos em serviços de saúde ${ }^{(8)}$.

O comportamento individual é um determinante relevante da vulnerabilidade, o que justifica focar as ações no indivíduo, embora isto não seja suficiente para o controle da situação. Consideramos que a vulnerabilidade do indivíduo em relação às PE (aerossóis, gotícula e contato) pode ser influenciada por fatores individuais como: conhecimento, percepção e até mesmo o engajamento.

O conhecimento dos indivíduos e o acesso à informação pode ser importante para reduzir a vulnerabilidade aos eventos adversos. Assim, é matéria de pesquisa quais elementos são necessários para elaboração do roteiro educacional e quais comportamentos e atitudes têm potencial para reduzir a vulnerabilidade individual aos eventos adversos.

Os indivíduos que se encontram em PE apresentam duas perspectivas de vulnerabilidade. Uma delas envolve o indivíduo e é decorrente de uma possível assistência inadequada. A outra envolve os demais indivíduos ao seu redor; esta sendo a potencialidade para a transmissão cruzada. $\mathrm{O}$ acesso à informação adequada pode reduzir a vulnerabilidade individual aos eventos adversos e contribuir para minimizar a vulnerabilidade dos outros pacientes. 
Acreditamos que o conceito de vulnerabilidade pode servir como um potente quadro referencial teórico para a elaboração de um roteiro que instrumentalize os PAS para contribuir na redução da vulnerabilidade individual. É importante destacar que, embora existam alguns materiais de orientação sobre as precauções voltadas ao público em geral ${ }^{(9)}$, nenhum instrumento de orientação utilizando este referencial teórico foi localizado em publicações. Além disto, tais materiais limitam-se a fornecer informação aos indivíduos, sem considerar suas percepções e crenças.

O objetivo do presente estudo foi elaborar e validar o conteúdo de um roteiro contendo elementos essenciais para orientação sobre PE para indivíduos adultos, tendo como quadro de referência o conceito de vulnerabilidade.

\section{MÉTODO}

Trata-se de um estudo do tipo metodológico, pois tratou do desenvolvimento e validação de instrumento ${ }^{(10)}$. $O$ estudo foi aplicado em três fases sequenciais: 1) captação da percepção de indivíduos em situação de PE, por meio da aplicação de um questionário; 2) elaboração de roteiro para orientação e 3) validação desse roteiro por especialistas. Uma representação esquemática do percurso metodológico é apresentada na Figura 1.

O estudo foi desenvolvido no município de São Paulono período de maio a julho de 2015. A captação da percepção dos indivíduos foi realizada por meio decoleta de dados em dois serviços de saúde com perfis assistenciais distintos. O serviço A caracteriza-se como hospital privado, presta assistência com financiamento de fundo privado (convênios e particulares). O serviço B caracteriza-se como hospitalescola, presta assistência como hospital de nível de atenção secundário, com atendimento integral com financiamento público pelo Sistema Único de Saúde (SUS). O estudo foi realizado na clínica médica e cirúrgica, em ambos os serviços.

Fase 1. O objetivo desta fase foi coletar informações para identificar conhecimentos de indivíduos internados em situação de PE nos respectivos hospitais sobre suas necessidades de saber sobre o tema, por meio da aplicação de questionário estruturado. O questionário foi aplicado pela pesquisadora principal e uma enfermeira treinada para esta finalidade. A fim de evitar a propagação de microrganismos, a coleta de dados realizou-se seguindo as recomendações de paramentação de acordo com as normas das Comissões de Controle de Infecção Hospitalar (CCIH) das instituições em questão.

Sujeitos do estudo na fase 1: O estudo teve como público alvo a população de indivíduos adultos em PE. Os critérios de inclusão foram: adultos, hospitalizados e em PE no momento da coleta de dados. Os critérios de exclusão foram: internados em Unidade de Terapia Intensiva (UTI), não orientados, não responsivos ou com comprometimento da sua capacidade cognitiva; em fenômenos depressivos; ou outras condições que contraindicassem a abordagem em forma de entrevista.

Fase 2. Nesta fase ocorreu a elaboração do roteiro com elementos essenciais para educação de indivíduos adultos em $\mathrm{PE}$, com base nos resultados obtidos na Fase 1 e na revisão de literatura ${ }^{(8)}$. $\mathrm{O}$ roteiro foi elaborado tendo como diretriz apoiar a orientação dos PAS aos indivíduos em PE com o objetivo de reduzir a vulnerabilidade individual aos eventos adversos. O conceito de vulnerabilidade foi utilizado como referencial teórico na elaboração do instrumento, dando foco ao acesso à informação e levando em consideração a experiência dos indivíduos em relação as PE.

Fase 3. O objetivo dessa fase foi validação de conteúdo do roteiroeducacional, com consulta a especialistas, tanto na perspectiva técnica no que se refere às PE como na perspectiva do conceito de vulnerabilidade. Os grupos de especialistas foram formados por enfermeiros, com experiência clínica e conhecimentos sobre PE e especialistas que possuem reconhecido saber quanto ao referencial teórico de vulnerabilidade. Foram convidados a participar de cada etapa de validação, por meio de carta convite, 12 especialistas em PE e 11 especialistas em vulnerabilidade, podendo ser considerado aceitável a desistência ao longo desta fase de até quatro profissionais em cada etapa.

A primeira rodada foi de validação de conteúdo pelos especialistas em PE, que avaliaram o roteiro referente o seu conteúdo, analisando a clareza, pertinência, objetividade, abrangência e representatividade. Na segunda rodada, o roteiro ajustado a partir destas considerações foi submetido 


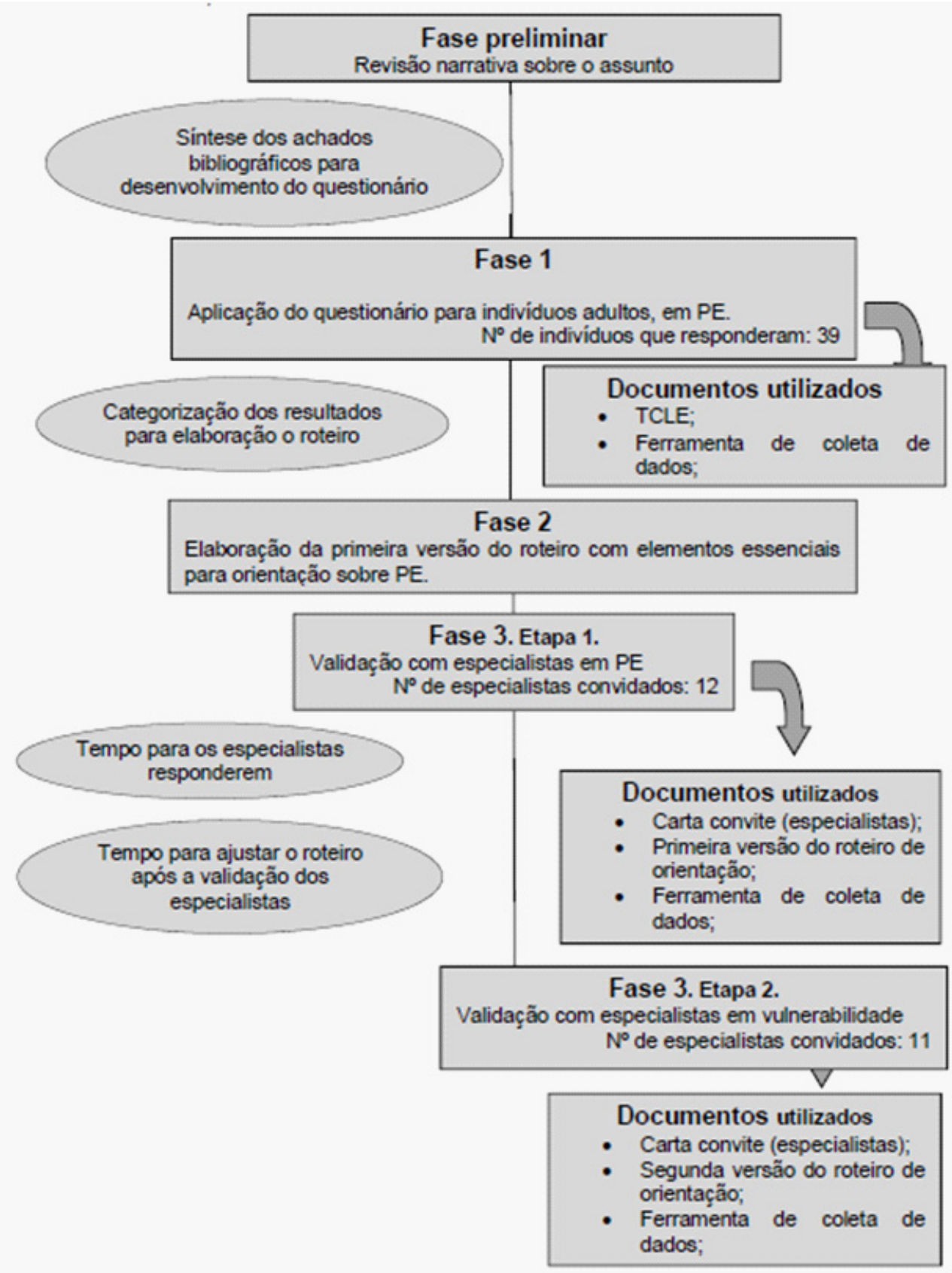

Figura 1 - Representação esquemática do percurso metodológico do estudo "Precauções específicas para evitar a transmissão de microrganismos: elaboração e validação de roteiro para contribuir na redução da vulnerabilidade individual". São Paulo, 2015

aos especialistas em vulnerabilidade, que validaram o roteiro referente a perspectiva da vulnerabilidade, verificando se o mesmo tem potencial para reduzir a vulnerabilidade individual para eventos adversos dos indivíduos em precauções específicas. Nesta fase os especialistas poderiam sugerir a retirada, acréscimo ou modificação dos itens.

Para validação de conteúdo foi utilizada escala do tipo likert com quatro opções de resposta: (1) discordo totalmente, (2) discordo, (3) concordo, (4) concordo totalmente. Foi utilizado o Índice de Validade de Conteúdo (IVC), que mede a porcentagem de juízes que concordaram sobre determinado aspecto do instrumento. O escore foi calculado por meio da soma de concordância das respostas 1 e 2 marcados pelos especialistas. O IVC, segundo a literatura, deve ser de 0,75 a 0,9 de concordância em cada item ${ }^{(10-11)}$. Para o presente estudo foram consideradas validadas as assertivas do roteiro que apresentaram um IVC de 0,75 .

Este projeto foi submetido e aprovado pelo Comitê da Escola de Enfermagem da Universidade de São Paulo sob parecer n ${ }^{\circ}$ 41497015.3.0000.5392. 
Fase 1. Dos 39 indivíduos em PE que responderam a entrevista, 25 (64,1\%) foram realizadas no serviço A e $14(35,9 \%)$ no serviço B. As entrevistas foram realizadas em média após onze dias de internação (variação de 1 a 87) e sete dias após a instituição de PE (variação de 0 a 73). Todos os indivíduos entrevistados estavam em quarto individual. As características dos indivíduos de cada cenário são apresentadas na Tabela 1. A média de anos de estudo foi de 14 anos no serviço particular e 7 anos no público e a média de idade foi respectivamente de: 44 e 52 anos.

Tabela 1 - Caracterização dos indivíduos adultos em precauções específicas segundo unidade hospitalar ( $\mathrm{n}=39$ ). São Paulo, SP, Brasil, 2015

\begin{tabular}{lcccc} 
& \multicolumn{2}{c}{ Serviço A } & \multicolumn{2}{c}{ Serviço B } \\
\hline Variável & $\mathbf{n}^{\mathbf{0}}$ & $\mathbf{( \% )}$ & $\mathbf{n}^{\mathbf{0}}$ & $\mathbf{( \% )}$ \\
\hline Gênero & & & & \\
\hline Feminino & 12 & 48 & 6 & 42 \\
\hline Masculino & 13 & 52 & 8 & 57 \\
\hline Unidade de internação & & & & \\
\hline Clínica médica & 19 & 76 & 8 & 57 \\
\hline Clínica cirúrgica & 3 & 12 & 6 & 42 \\
\hline Precaução instituída & & & & \\
\hline Documentada em prontuário & 15 & 60 & 10 & 71 \\
\hline Não documentada & 10 & 40 & 4 & 28
\end{tabular}

Os indivíduos que estavam em PE para contato eram 32(82\%), as PE para gotícula e mistas (contato + gotícula ou contato + aerossóis) representaram um (2,5\%) cada e as PE para aerossóis foram quatro $(10,2 \%)$.Verificou-se que as PE para contato foram instituídas por colonização de agentes multirresistente em $14 \%$ das vezes, por infecção em $53 \%$ e por investigação de multirresistentes em $32 \%$ das vezes.

As informações sobre o conhecimento dos indivíduos a cerca das precauções específicas estão sumarizadas na Tabela 2.

Tabela 2 - Principais informações sobre o conhecimento dos indivíduos acerca das precauções específicas ( $\mathrm{n}=39$ ). São Paulo, SP, Brasil, 2015

\begin{tabular}{lc} 
Questões & Sim n(\%) \\
\hline Sabe por que foi hospitalizado? & $37(94,8)$ \\
\hline Sabe se o diagnóstico necessita de algum cuidado específico? & $19(48,7)$ \\
\hline Sabe como transmite a sua doença? & $17(43,5)$ \\
\hline Os profissionais orientaram sobre o motivo de utilizarem EPIs*? & $26(66,6)$ \\
\hline Recebeu informações sobre Higiene de Mãos? & $16(41)$ \\
\hline Acredita que pode receber visitas normalmente, sem nenhuma restrição? & $38(97,4)$ \\
\hline $\begin{array}{l}\text { Os familiares e visitantes receberam algum orientação de como agir em relação as precauções } \\
\text { específicas? }\end{array}$ & $17(42,8)$ \\
\hline Já ficou internado anteriormente em precauções específicas? & $15(38,4)$
\end{tabular}

*EPIs: Equipamentos de proteção individual

A percepção dos entrevistados em relação à frequência de uso de EPIs pelos PAS é apresentada na Tabela 3. Os pacientes indicaram a sua percepção da frequência de uso de todos os EPIs, inclusive aqueles utilizados em PP.

A percepção dos entrevistados em relação à educação em saúde recebida é sumarizada na Tabela 4. 
Tabela 3 - Uso de equipamento de proteção individual pelos profissionais segundo a percepção dos indivíduos entrevistados e o tipo de precaução específica (n=39). São Paulo, SP, Brasil, 2015

\begin{tabular}{|c|c|c|c|c|c|c|}
\hline \multirow{2}{*}{$\begin{array}{l}\text { Tipo de } \\
\text { PE** }\end{array}$} & \multirow{2}{*}{$\begin{array}{c}\text { Total de paciente } \\
\text { em PE** }\end{array}$} & \multirow[t]{2}{*}{ EPIs* } & \multicolumn{4}{|c|}{ Frequência segundo a percepção do paciente } \\
\hline & & & Sempre & Às vezes & Em procedimentos & Nunca \\
\hline \multirow[t]{4}{*}{ Aerossóis } & \multirow[t]{4}{*}{4} & Avental & 0 & 0 & 0 & 4 \\
\hline & & Luvas & 2 & 0 & 1 & 1 \\
\hline & & Máscara comum & 0 & 0 & 0 & 4 \\
\hline & & Máscara N-95 & 4 & 0 & 0 & 0 \\
\hline \multirow[t]{4}{*}{ Contato } & \multirow[t]{4}{*}{32} & Avental & 20 & 4 & 6 & 2 \\
\hline & & Luvas & 20 & 4 & 8 & 0 \\
\hline & & Máscara comum & 1 & 2 & 7 & 22 \\
\hline & & Máscara N-95 & 0 & 0 & 0 & 32 \\
\hline \multirow[t]{4}{*}{ Gotícula } & \multirow[t]{4}{*}{1} & Avental & 0 & 0 & 0 & 1 \\
\hline & & Luvas & 0 & 0 & 1 & 0 \\
\hline & & Máscara comum & 1 & 0 & 0 & 0 \\
\hline & & Máscara N-95 & 0 & 0 & 0 & 0 \\
\hline \multirow{4}{*}{$\begin{array}{l}\text { Contato + } \\
\text { Aerossóis }\end{array}$} & \multirow[t]{4}{*}{1} & Avental & 0 & 1 & 0 & 0 \\
\hline & & Luvas & 1 & 0 & 0 & 0 \\
\hline & & Máscara comum & 0 & 0 & 0 & 1 \\
\hline & & Máscara N-95 & 1 & 0 & 0 & 0 \\
\hline \multirow{4}{*}{$\begin{array}{l}\text { Contato + } \\
\text { Gotícula }\end{array}$} & \multirow[t]{4}{*}{1} & Avental & 0 & 1 & 0 & 0 \\
\hline & & Luvas & 1 & 0 & 0 & 0 \\
\hline & & Máscara comum & 1 & 0 & 0 & 0 \\
\hline & & Máscara N-95 & 0 & 0 & 0 & 0 \\
\hline
\end{tabular}

*EPIs: Equipamentos de proteção individual; **PE: Precaução específica. Nota: itens em negrito se referem aos EPI requeridos para cada PE segundo a norma da $\mathrm{CCIH}$.

Tabela 4 - Informações fornecidas pelos profissionais aos indivíduos em precauções específicas sobre o uso de equipamento de proteção individual (n=26). São Paulo, SP, Brasil, 2015

\begin{tabular}{lcc} 
Variáveis & $\mathbf{n}^{\mathbf{0}}$ & $\mathbf{\%}$ \\
\hline O que foi explicado? & & \\
\hline O que usar & 15 & 57,6 \\
\hline Quando usar & 10 & 38,4 \\
\hline Como usar & 15 & 57,6 \\
\hline O que acontece se não usar & 18 & 69,2 \\
\hline Qual profissional fez a orientação? & 10 & \\
\hline Auxiliar/técnico de enfermagem & 18 & 69,4 \\
\hline Enfermeiro & 10 & 38,4 \\
\hline Médico & & \\
\hline Quando foi a orientação? & 12 & 46,1 \\
\hline No momento da internação & 12 & 46,1 \\
\hline Após a instituição da precaução específica & 1 & 3,8 \\
\hline Após questionar um profissional & & \\
\hline Como foi a orientação? & 24 & 92 \\
\hline Apenas falada & 2 & 7,6
\end{tabular}


Os entrevistados consideraram que sabem o suficiente sobre as PE em 16 (41\%) dos casos, 16 (41\%) apontaram saber parcialmente e sete $(17,9 \%)$ disseram não ter nenhuma informação sobre o assunto. Os entrevistados foram questionados sobre o comportamento em relação a $\mathrm{PE}$, considerando crenças e sentimentos. Os entrevistados acreditavam que as medidas de PE adotadas protegem a si próprio26 (66,6\%), a sua família29 (74,3\%) e aos outros pacientes 32 (82\%). Quanto aos hábitos pessoais de higiene de mãos, 37 (94,8\%) dos indivíduos referiram higienizar as mãos após usar o banheiro e 30 (79,4\%) antes de se alimentar.

Três $(7,6 \%)$ dos entrevistados acreditavam que os PAS entravam menos vezes no quarto devido a PE. Os entrevistados perceberam que os PAS ficavam o mesmo tempo no quarto de PE em 19 (48,7\%), e não notaram diferença em 17 (43,5\%) das vezes. Apenas um (2,5\%) acreditava que o PAS ficavam mais tempo no quarto devido às PE e dois acreditavam que ficavam menos tempo pelo mesmo motivo. Sobre o uso de EPIs por parte da equipe, 32(82\%) dos entrevistados acreditavam que todos os profissionais deveriam utilizar os EPIs ao entrar no quarto ou em contato com o leito. Apesar disto, 12 (84\%) não fizeram nada quando a equipe não se paramentava e apenas um questionou o profissional que não utilizou os EPIs. Outro paciente orientou para que utilizassem; um paciente não soube como abordar o profissional e tiveram receio da reação do profissional. Dos indivíduos que necessitavam sair do quarto para algum procedimento, cinco $(45,4 \%)$ relataram não utilizar nenhuma medida adicional.

Os sentimentos relacionados à situação de PE foram em sua majoritariamente positivos, como: bem cuidado $(n=28 ; 74,3 \%)$ e seguro $(n=23 ; 61,5 \%)$, foram citados também por um entrevistado o aumento do conforto e da privacidade. Os sentimentos negativos tiveram menor impacto: ansioso( $\mathrm{n}=7 ; 17,9 \%)$, com raiva $(n=1 ; 2,5 \%)$, com sentimento de culpa $(n=3 ; 7,6 \%)$, solitário( $n=6 ; 15,3 \%)$, estressa do $(n=7$; $17,9 \%)$ e com medo ( $n=9 ; 23,0 \%)$. Outros sentimentos negativos foram citados pelos entrevistados, respectivamente uma citação para cada: magoado, triste, constrangido, confuso, nervoso, preocupado e descrente da situação de PE.

Fase 2. Os elementos supra citados apoiaram a construção do roteiro educativo. Com o objetivo de obter uma melhor organização do roteiro, optou-se por apresentá-lo em três tópicos: Quando abordar o paciente, Como abordar o paciente, Acompanhamento e avaliação.

Fase 3. Participaram do estudo enfermeiras, com experiência profissional em controle de infecção e docência/pesquisa, com uma média de 17 anos de formação.

Na primeira rodada, todos os especialistas avaliaram o material quanto a clareza na primeira leitura. A informação foi considerada pertinente (75\%) e relevante $(100 \%)$ para apoiar os PAS na elaboração de ações educativas para indivíduos adultos em PE. Os atributos de abrangência e representatividade no universo das PE obtiveram $75 \%$ e $87 \%$ de concordância, respectivamente.

Na segunda rodada referente ao conceito de vulnerabilidade, nove especialistas retornaram suas contribuições.Participaram do estudo enfermeiras, com atuação profissional em docência/pesquisa, saúde laboral e gestão de serviços, com uma média de 28 anos de formação. Para esses especialistas, o material foi avaliado claro na primeira leitura, a informação foi considerada pertinente (88\%) e relevante $(100 \%)$ para conduzir PAS na elaboração de ações educativas para indivíduos adultos em PE. Os atributos de abrangência e representatividade no universo da vulnerabilidade individual obtiveram $66,6 \%$ e $77 \%$ de concordância, respectivamente.

Com o intuito de alcançar o máximo de concordância possível, o processo de validação sofreu um procedimento adicional por meio de entrevistas pessoais com duas especialistas a fim de obter melhor ajuste da ferramenta, após esta etapa o atributo de abrangência recebeu IVC de $77 \%$ de concordância.

\section{DISCUSSÃO}

Este estudo apresenta uma proposta de roteiro para apoiar a elaboração de ações educativas, que visa a contribuir para a transformação das relações atuais entre enfermeiros e os usuários do serviço de saúde, na medida em que oferta uma ferramenta que favorece a autonomia e o engajamento dos indivíduos.

Por meio dos relatos da percepção dos indivíduos a adesão às precauções pelos PAS, verificou-se 
falhas na adesão a PE para contato, o que também foi observado em outros estudos como relacionado com uma limitação de comportamento e percepção dos PAS $^{(1)}$.

Alguns autores discutem que os enfermeiros não tem uma visão integral do indivíduo como agente de sua própria recuperação. Além disso, descrevem que os PAS não conhecem ao certo a expressão "educação em saúde", confundindo-a com educação continuada; apesar disto, os PAS demonstram interesse em exercer alguma atividade de educação de indivíduos. Portanto, parece que os PAS necessitam de mais informações sobre o tema para que possam se sensibilizar e praticar a educação em saúde de qualidade ${ }^{(12)}$.

Conforme apresentado nos resultados, houve diferença entre o nível de escolaridade entre os locais de coleta de dados. Um autor aponta que alguns fatores, como o grau de escolaridade, podem influenciar na compreensão das informações e tomada de atitude dos indivíduos ${ }^{(13)}$. O roteiro elaborado propõe um resgate aos saberes dos indivíduos antes da introdução de conhecimentos novos, levando em conta estes potenciais fatores.

Segundo uma revisão realizada sobre a eficácia da participação dos pacientes, a participação e envolvimento do indivíduo nos cuidados de saúde tem recebido grande importância na minimização de eventos adversos ${ }^{(14)}$. Entretanto, apesar dos indivíduos entrevistados na presente pesquisa relatarem estar conscientes do motivo da sua internação, metade referiu não ter recebido orientações sobre as PE, apontando não ter conhecimento suficiente sobre as PE. Isto aponta para uma maior necessidade de informação e apropriação da sua situação por parte dos indivíduos. Ainda, segundo outros autores o familiar pode colaborar com a minimização de eventos adversos, devendo ser envolvido nas ações educativas $^{(2,15)}$.

Em relação ao comportamento, identificou-se que existe uma percepção de que as PE protegem a si próprio e aos outros ao redor. Apesar disto, os indivíduos relatam não se manifestar quando algum PAS toma atitude incorreta quanto ao uso de EPIs. Isto pode se dar devido à dificuldade de diálogo entre as partes envolvidas ou temor por parte do indivíduo de que este tipo de abordagem afete os cuidados que irá receber.

O comportamento protetor vai ao encontro do desafio do diálogo entre PAS e indivíduos em internação hospitalar, envolvendo fatores relacionados a ambos. Dentre eles, a falta de aceitação do indivíduo em desempenhar um novo papel, que passa ser o principal responsável por sua saúde e segurança. Já os PAS tem a tendência a manter o controle, resistindo ao envolvimento do indivíduo na decisão do cuidado. Outras vezes os PAS alegam falta de tempo, não ter conhecimento suficiente e não saber lidar com a situação ${ }^{(16-17)}$.

Esta dificuldade de comunicação pode ser superada através do diálogo e do uso do roteiro aqui apresentado, contendo elementos essenciais para a orientação de indivíduos em PE a fim de reduzir a vulnerabilidade individual aos eventos adversos através da superação do modelo de educação impositiva.

A educação em saúde corrente é baseada apenas na prática de uma educação modeladora, onde são passadas para os indivíduos informações prescritivas sem levar em consideração suas experiências. O modelo de educação dialógica propõe uma comunicação entre educando e educador; esta dimensão da educação pressupõe que cada indivíduo é a interação de tudo ao seu redor ${ }^{(18)}$. Este mesmo autor diz que a atitude construtivista é o melhor meio para que os indivíduos busquem informações que façam sentido para eles e que através disto encontrem formas de superar as situações que aumentam a vulnerabilidade.

Os resultados demonstraram que a maioria dos indivíduos entrevistados demonstrava sentimentos positivos em relação à situação de $\mathrm{PE}$, em contradição a outro estudo que apresenta um impacto negativo no bem estar psicológico, segurança e satisfação relacionada a estes indivíduos ${ }^{(19)}$.

O roteiro aqui apresentado foi elaborado a partir das percepções dos indivíduos sob PE e embasado no referencial teórico de vulnerabilidade. Acreditamos que esta ferramenta terá potencial para dar suporte às ações educativas dos PAS no que tange às PE. Entretanto, para o utilização e sucesso do roteiro é imprescindível que os PAS estejam sensibilizados quanto a importância da educação do indivíduo que está sob seus cuidados. Portanto, é importante destacar esta limitação da ferramenta, 
que não foi desenvolvida com o propósito de atuar na sensibilização destes profissionais.

Na validação de conteúdo os especialistas sugeriram direcionar o roteiro para enfermeiros, porém os indivíduos entrevistados apontam a participação de outros profissionais no momento da orientação sobre PE. Por este motivo optamos por estender o escopo do roteiro educacional para todas as categorias de PAS.

O estudo utilizou como método de abordagem a percepção dos indivíduos através de um questionário quantitativo, o que impõe algumas limitações no que tange a captação de percepções humanas. Contudo, o objetivo principal desta etapa foi captar os elementos-chave a serem incorporados em um projeto educativo, não sendo proposto adensar no conhecimento destas percepções.

Etapas subsequentes deste projeto estão sendo delineadas, incluindo a aplicação deste roteiro, avaliação de sua factibilidade e impactos na prática assistencial com vistas a minimização de eventos adversos.

\section{- CONCLUSÃO}

Considera-se que o roteiro proposto neste estudo é um avanço no que diz respeito a ferramentas de apoio para educação de indivíduos em PE, pois abordou aspectos fundamentais para o diálogo entre o indivíduo e PAS, por meio do referencial teórico do conceito de vulnerabilidade, tendo sido validado por especialistas quanto ao seu conteúdo.O roteiro elaborado através deste estudo é uma ferramenta que irá contribuir para embasar as ações de enfermagem na assistência aos pacientes em $\mathrm{PE}$, favorecendo as práticas de educação em saúde.

\section{REFERÊNCIAS}

1. de Oliveira AC, Cardosoll CS, Mascarenhas D. Conhecimento e comportamento dos profissionais de um centro de terapia intensiva em relação à adoção das precauções de contato. Rev. Latino-Am. Enfermagem. [Internet]2009;17(5)[acesso em 10 mai 2013]. Disponível: http://dx.doi.org/10.1590/S0104-11692009000500005.

2. Rabelo AHS, de Souza TV. O conhecimento do familiar/acompanhante acerca da precaução de contato: Contribuições para a enfermagem pediátrica. Esc. Anna Nery. [Internet]2009;13(2) [acesso em10 mai 2013]. Disponível: http://dx.doi.org/10.1590/S1414-81452009000200006.

3. Cardim MG, dos Santos AEV, Nascimento MAL, Biesbroek FCC. Crianças em isolamento hospitalar: relações e vivências com a equipe de enfermagem. RevEnferm UERJ. 2008;16(1):32-8.

4. Morgan DJ, Pineles L, Shardell M, Grahan MM, Mohammadi S, Forrest GN, et al. The effect of contact precautions on healthcare worker activity in acute care hospitals. Infect Control HospEpidemiol. 2013;34(1):69-73.

5. Bertolozzi MR, Nichiata LYI, Takahashi RF, Ciosak SI, Hino P, do Val LF,et al. Os conceitos de vulnerabilidade e adesão na Saúde Coletiva. Rev. esc. enferm. USP.[Internet]2009;43(n.esp2)[acesso em 27 jul2013]. Disponível: http://dx.doi.org/10.1590/S0080-62342009000600031.

6. Meyer DEE, de Mello DF, Valadão MM, Ayres JRCM. "Você aprende. A gente ensina?"Interrogando relações entre educação e saúde desde a perspectiva da vulnerabilidade. Cad. Saúde Pública. [Internet]2006;22(6) [acesso em 06 ago 2013]. Disponível: http://dx.doi.org/10.1590/S0102-311X2006000600022.

7. Nunes L. Usuários dos Serviços de Saúde e os seus direitos. RevBras Bioética. 2006;2(2):201-19.

8. Juskevicius LF, Padoveze MC. Vulnerabilidade dos pacientes quanto às precauções específicas para doenças infecciosas. Revenferm UFPE online. No prelo 2016.

9. da Silva PF, Padoveze MC. Infecções relacionadas a serviços de saúde, orientação para o público em geral: Conhecendo um pouco mais sobre as Precauções Específicas. Centro de Vigilância Epidemiológica; 2012.p.1-9.

10. Polit D, Beck CT. Fundamentos de pesquisa em enfermagem:métodos, avaliação e utilização. $7^{a}$ ed. Porto Alegre: Artmed; 2011. 
11. Alexandre NMC, Coluci MZO. Validade de conteúdo nos processos de construção e adaptação de instrumentos de medidas. Ciênc. saúde coletiva. 2011;16(7):3061-8.

12. Olivi M, Oliveira MLF Educação para saúde em unidade hospitalar: um espaço profissional do enfermeiro. 2003;2(2):131-8.

13. Kistin CJ. Patient health literacy and the practice of evidence-based medicine. EvidBased Med. [Internet] 2012;17(5) [acesso em04 mai 2013]. Disponível: http://dx.doi.org/10.1136/ebmed-2012-100712.

14. Logtin Y, Sax H, Leape LL, Sheridan SE, Donaldson L, Pittet D. Patient Participation: current knowledge and applicability to patient safety. Mayo Clin Proc. 2010;85(1):53-62.

15. de Morais IM. Vulnerabilidade do doente versus autonomia individual. Rev. Bras. Saúde Mater. Infant.[Internet]2010;10(Suppl.2)[acesso em04 dez 2013].Disponível: http://dx.doi.org/10.1590/S151938292010000600010 .

16. Guez VMA. Percepções do familiar da criança com germe multirresistente sobre medida de precaução de contato [monografia].Porto Alegre (RS):Universidade Federal do Rio Grande do Sul;2009.

17. Melo G, dos Santos RM, Trezza MCSF. Entendimento e prática de ações educativas de profissionais do Programa Saúde da Família de São Sebastião-AL: detectando dificuldades. Rev. bras. enferm. 2005;58(3):290-5.

18. Ayres JRCM. Práticas educativas e prevenção de HIV/Aids: lições e desafios atuais. Interface, Comun., Saúde, Educ. 2002;6(11):11-24.

19. Abad C, Fearday A, Safdar N. Adverse effects of isolation in hospitalised patients: a systematic review. J Hosp Infect.[Internet]2010;76(2) [acessoem 04 mai 2013]. Disponível: http://dx.doi.org/10.1016/j.jhin.2010.04.027. 